\title{
Effet de diverses conditions de traitement à l'ammoniac d'une paille de blé sur les teneurs en acides $p$-coumarique et férulique et sur la degradabilité de l'azote mesurée « in situ »
}

\author{
Rabéha CHABACA ${ }^{\mathrm{a}}$, Aristide LARWENCE ${ }^{\mathrm{b} *}$, Michel PAYNOT $^{\mathrm{c}}$, \\ Jean-Louis TISSERAND ${ }^{\mathrm{d}}$ \\ a INA, Dpt de Zootechnie, 16200 El-Harrach, Algérie \\ b ENSIA-SIARC, B.P. 5098, 34033 Montpellier Cedex, France \\ ${ }^{c}$ INRA, B.V. 1540, 21034 Dijon Cedex, France \\ d ENESAD, B.P. 1607, 21036 Dijon Cedex, France
}

(Reçu le 21 juillet 1998 ; accepté le 9 décembre 1999)

\begin{abstract}
Effect of treatment of wheat straw with ammonia under different conditions on $p$-coumaric and ferulic acid content and on "in situ" nitrogen degradability. The main aim of our work was to study under various conditions, the effects of ammonia-treated wheat straw on $p$-coumaric ApC) and ferulic acid content (AF) as well as the nitrogen degradability (dtN) and their relation to understand the abnormally high amounts of faecal nitrogen in the ruminant fed ammonia treated straw. 16 straws were submitted to different conditions of ammonia ( 3 and 5\%), duration $\left(15,30,45\right.$ and 75 days) and temperature $\left(15\right.$ and $\left.35^{\circ} \mathrm{C}\right)$. Ammoniac increased the nitrogen matter from 2.1 to $11.3 \%$ of dry matter (DM). The FA and ApC contents were respectively $3.89 \mathrm{~g}$ and $2.94 \mathrm{~g} / \mathrm{kg}$ DM for untreated straw (PNT). After treatment, FA and ApC content decreased respectively of 7 and $22 \%(P<0.05$ and $P<0.001)$. The degradability of PNT is very low (15\%). It increased with the treatment, particularly with the temperature: $63 \%$ at $15{ }^{\circ} \mathrm{C}$ and $71 \%$ at $35{ }^{\circ} \mathrm{C}$. This result is explained by the increase of model parameters a $(+15 \%)$ and c $(+27 \%)$. The correlation established between dtN, kinetic points of nitrogen degradation and FA, ApC and FA + ApC were low. They were only high $(P<0.001)$, during the first $8 \mathrm{~h}$ of incubation. 30 to $37 \%$ of the nitrogen degradability were explained by FA and ApC respectively. It is concluded that neither FA and ApC contents nor an irreversible fixation of nitrogen of the treatment on the straw can explain satisfactorily the abnormally high amounts of faecal nitrogen excretion observed in ruminants fed ammoniatreated straw. The synthesis and the release of indigestible nitrogenous compounds, probably made of nitrogen and phenolic acids, explain this phenomenon indeed these compounds could capture in an irreversible way a part of the nitrogen of treatment.
\end{abstract}

straw / ammonia treatment / "in situ” nitrogen degradability / phenolic acids

* Correspondance et tirés à part

E-mail: larwence@ siarc.cnearc.fr 
Résumé - L'objectif de ce travail a été d'étudier l'effet de différentes conditions de traitement d'une paille de blé à l'ammoniac sur la dégradabilité théorique de l'azote $(\mathrm{dtN})$, la teneur en acides $p$-coumarique (ApC) et férulique (AF) ainsi que les relations entre la dtN et ces deux acides. $\mathrm{Au}$ total, 16 lots de pailles (PT) ont été traités en combinant trois paramètres : dose d'ammoniac ( 3 et $5 \%$ ), température $\left(15\right.$ et $\left.35^{\circ} \mathrm{C}\right)$ et durée de traitement $(15,30,45$ et 75 jours $)$. Le traitement à l'ammoniac a augmenté logiquement, la teneur en matières azotées des pailles qui est passée de 2,1 à $11,3 \%$ de la MS. En revanche, les teneurs en $\mathrm{ApC}$ et en $\mathrm{AF}$ ont diminué respectivement de $7 \%(p<0,05)$ et de $22 \%(p<0,001)$. Le traitement a amélioré très fortement la dtN qui est passée de $15 \%$ (PNT) à 63 et $71 \%$ respectivement pour les PT à $15{ }^{\circ} \mathrm{C}$ et $35^{\circ} \mathrm{C}$; tout comme les paramètres a $(+15 \%)$ et c $(+27 \%)$ de la cinétique de dégradation. La dtN a été modérément affectée par la présence des deux acides phénoliques. Ainsi, seuls 30 à $37 \%$ de la dtN sont expliqués respectivement par $\mathrm{AF}$ et ApC. Ces effets se sont surtout manifestés durant, les premières heures d'incubation $(2-8 \mathrm{~h})$. L'ensemble de nos résultats montrent que ni les acides phénoliques, ni la fixation irréversible sur la paille de l'azote provenant du traitement n'explique de façon satisfaisante l'excrétion anormalement élevée d'azote chez les animaux consommant des pailles de blé traitées à l'ammoniac. La synthèse et la libération de composés azotés indigestibles constitués par l'azote et les acides phénoliques expliqueraient ce phénomène. En effet, ces composés pourraient emprisonner de façon irréversible une partie de l'azote provenant du traitement.

paille / traitement à l'ammoniac / dégradabilité « in situ » de l'azote / acides phénoliques

\section{INTRODUCTION}

Le traitement à l'ammoniac est un moyen bien connu pour améliorer la valeur alimentaire des pailles [32, 33]. Il associe les propriétés basiques de l'ammoniac à un apport d'azote pour les micro-organismes du rumen. Sur 46 expériences répertoriées dans la littérature [6], la digestibilité de la matière organique et les quantités de matière sèche ingérées progressent respectivement de 29 et de $23 \%$.

Néanmoins, l'utilisation par l'animal de l'azote apporté par le traitement et fixé par la paille est bien inférieure à celle théoriquement attendue, compte tenu de la teneur en azote des pailles (9 à $10 \%$ ) et de sa nature non protéique $[3,6]$.

Cette anomalie qui aboutit à une excrétion d'azote anormalement élevée chez l'animal, a été observée depuis un quart de siècle [29, 33]. Les chiffres rapportés pour les pailles du nord de l'Europe [6] indiquent que 25 à $41 \%$ de l'azote fixé après traitement, ne sont pas utilisés par l'animal.

Deux types d'explications peuvent être proposées :
- L'azote apporté par le traitement serait fortement fixé sur les parois de la paille. De ce fait, les résidus de paille chargés en azote moins bien dégradés dans le rumen seraient fermentés dans le gros intestin avec production d'azote microbien qui serait ensuite excrété dans les fèces [13, 20]. Cependant, cette hypothèse n'est pas vérifiée : certains auteurs [12, 27] indiquent en effet que 75 à $80 \%$ de l'azote apporté par le traitement sont dégradés dans le rumen. Il est par ailleurs montré [28], que la contribution de l'azote des fraction NDF ou ADF à l'augmentation de l'azote fécal est faible (16 à $20 \%$ ), tout comme celle de l'azote microbien (11 à $28 \%$ ). De même, le traitement à l'ammoniac ne modifie pas la quantité et le profil des acides aminés excrétés dans les fèces [24]. Une autre hypothèse peut donc être envisagée.

- Certains acides phénoliques notamment $p$-coumarique et férulique présents dans les pailles [9], auraient un effet dépressif sur les micro-organismes du rumen $[21,36,38]$ et un effet barrière et stéréochimique sur la dégradation de la paroi [4] se traduisant par une diminution de la croissance bactérienne 
et de l'activité cellulolytique d'où une dégradation moindre de l'azote. Celle-ci pourrait aussi être modifiée dans les pailles ammoniaquées en fonction des conditions de traitement compte tenu que le traitement à l'ammoniac diminue très significativement la teneur en acides $p$-coumarique et férulique $[11,25]$.

Dans ce travail, l'effet de diverses conditions de traitement (température, dose et durée) d'une paille de blé à l'ammoniac à été étudié d'une part sur la teneur en acides $p$-coumarique et férulique et d'autre part, sur la dégradabilité « in situ » de l'azote. Les relations entre ces différents critères sont examinées.

\section{MATÉRIEL ET MÉTHODES}

\subsection{Les pailles}

Une paille de blé dur récoltée sur la station de l'ITGC de l'Oued Smar dans la région d'Alger a été traitée à l'ammoniac selon la technique suivante : $2 \mathrm{~kg}$ de paille grossièrement hachée (brins de $8 \mathrm{~cm}$ environ) ont été introduits dans un sac en plastique noir résistant. L'ammoniac produit par chauffage d'ammoniaque liquide a été lentement injecté dans les sacs. L'opération terminée, les sacs ont été hermétiquement fermés. Au total, 16 lots de paille ont ainsi été préparés (Tab. I) selon différentes

Tableau I. Identification et composition chimique des pailles (\% MS).

\begin{tabular}{|c|c|c|c|c|c|c|c|c|c|c|c|}
\hline \multicolumn{4}{|c|}{ Identification } & \multicolumn{8}{|c|}{$\begin{array}{c}\text { Composition en matières azotées en parois } \\
\text { et en acides phénoliques }\end{array}$} \\
\hline Pailles & $\mathrm{T}$ & $\mathrm{D}$ & d & $\operatorname{MS}(\%)$ & MAT & NDF & $\mathrm{ADF}$ & $\mathrm{ADL}$ & $\mathrm{ApC}$ & $\mathrm{AF}$ & $\mathrm{ApC}+\mathrm{AF}$ \\
\hline PT1 & 15 & 3 & 15 & 92,4 & 9,1 & 79,1 & 50,4 & 7,3 & 3,07 & 3,84 & 6,91 \\
\hline PT2 & 15 & 3 & 30 & 92,8 & 8,6 & 79,6 & 50,2 & 7,6 & 2,63 & 3,41 & 6,04 \\
\hline PT3 & 15 & 3 & 45 & 92,9 & 8,7 & 80,8 & 53,2 & 8,0 & 3,01 & 3,48 & 6,49 \\
\hline PT4 & 15 & 3 & 75 & 92,0 & 8,6 & 79,1 & 54,3 & 9,0 & 2,99 & 3,59 & 6,58 \\
\hline PT5 & 15 & 5 & 15 & 90,8 & 8,3 & 79,6 & 52,4 & 8,1 & 2,95 & 3,79 & 6,74 \\
\hline PT6 & 15 & 5 & 30 & 89,9 & 9,6 & 80,2 & 54,5 & 8,6 & 3,01 & 3,26 & 6,27 \\
\hline PT7 & 15 & 5 & 45 & 90,3 & 9,1 & 78,1 & 51,9 & 7,7 & 2,70 & 2,75 & 5,45 \\
\hline PT8 & 15 & 5 & 75 & 89,9 & 10,7 & 78,5 & 53,2 & 8,3 & 2,79 & 2,82 & 5,61 \\
\hline PT9 & 35 & 3 & 15 & 90,9 & 11,1 & 78,9 & 45,5 & 6,2 & 2,98 & 3,30 & 6,28 \\
\hline PT10 & 35 & 3 & 30 & 91,3 & 11,4 & 75,1 & 44,9 & 6,3 & 2,45 & 3,02 & 5,47 \\
\hline PT11 & 35 & 3 & 45 & 91,6 & 11,7 & 75,9 & 45,7 & 6,1 & 2,61 & 3,61 & 5,62 \\
\hline PT12 & 35 & 3 & 75 & 90,4 & 11,8 & 75,2 & 45,4 & 6,5 & 2,66 & 3,42 & 6,08 \\
\hline PT13 & 35 & 5 & 15 & 90,5 & 13,7 & 73,5 & 46,4 & 6,6 & 2,44 & 2,90 & 5,34 \\
\hline PT14 & 35 & 5 & 30 & 90,8 & 13,4 & 74,7 & 47,5 & 6,7 & 2,38 & 2,60 & 4,98 \\
\hline PT15 & 35 & 5 & 45 & 90,4 & 13,1 & 74,6 & 46,8 & 7,0 & 2,71 & 2,79 & 5.49 \\
\hline PT16 & 35 & 5 & 75 & 91,8 & 12,4 & 74,1 & 46,7 & 6,2 & 2,51 & 2,59 & 5,10 \\
\hline PNT & - & - & - & 91,7 & 2,2 & 78,2 & 45,4 & 6,1 & 2,94 & 3,89 & 6,83 \\
\hline Effet $T$ & - & - & - & - & $* * *$ & $* * *$ & $* * *$ & $*$ & $*$ & $* * *$ & $*$ \\
\hline Effet D & - & - & - & - & $*$ & NS & NS & NS & NS & $* * *$ & NS \\
\hline Effet d & - & - & - & - & NS & NS & NS & NS & NS & $*$ & NS \\
\hline $\mathrm{T} \times \mathrm{D}$ & - & - & - & - & NS & NS & NS & NS & NS & $*$ & NS \\
\hline $\mathrm{T} \times \mathrm{d}$ & - & - & - & - & NS & NS & NS & NS & NS & $*$ & NS \\
\hline $\mathrm{D} \times \mathrm{d}$ & - & - & - & - & NS & NS & NS & NS & NS & $*$ & NS \\
\hline RSD & - & - & - & - & 0,88 & 0,89 & 1,11 & 0,42 & 0,18 & 0,07 & 0,39 \\
\hline $\mathrm{R}^{2}$ & - & - & - & - & 0,96 & 0,97 & 0,98 & 0,96 & 0,88 & 0,99 & 0,91 \\
\hline
\end{tabular}

$\mathrm{T}$ : température ; $\mathrm{D}:$ dose ; $\mathrm{d}$ : durée ; RSD : écart-type résiduel du modèle $; \mathrm{R}^{2}$ : taux de variation expliquée du modèle ; NS : non significatif ; $*: p<0,05 ; * * *: p<0,01$ à 0,001 . La PNT, n'est pas prise en compte dans l'analyse de variance. La MS est dosée, immédiatement à la sortie des sachets. 
combinaisons de doses ( 3 et $5 \%$ d'ammoniac), température $\left(15\right.$ et $\left.35^{\circ} \mathrm{C}\right)$ et durée de traitement $(15,30,45$ et 75 jours). En hiver, les sacs à $15{ }^{\circ} \mathrm{C}$ ont été conservés dans un local où la température a varié entre 12 et $17^{\circ} \mathrm{C}$; les sacs à $35^{\circ} \mathrm{C}$ ont été mis à l'étuve réglée à cette température. À l'échéance de chaque temps de traitement, la paille a été extraite des sacs et un échantillon représentatif de $50 \mathrm{~g}$ a été prélevé pour mesurer la MS ; le reste a été aéré pendant $48 \mathrm{~h}$ à température ambiante puis broyé à la grille de $0,8 \mathrm{~mm}$ et conservée pour mesures et analyses.

\subsection{Méthodes analytiques}

Sur les échantillons broyés ont été déterminées la teneur en matières azotées totales (MAT) et en sortie de sac, celle en matière sèche (MS), selon les méthodes de l'AOAC [2]. La paroi totale (NDF), la lignocellulose (ADF) et la lignine brute (ADL) ont été analysées selon la méthode séquentielle de VanSoest [34].

Les acides phénoliques : $p$-coumarique (ApC)et férulique (AF) ont été extraits par hydrolyse basique à chaud $\left(70{ }^{\circ} \mathrm{C}\right)$ pendant 3 heures à l'obscurité, à partir d'un échantillon de $100 \mathrm{mg}$ de paille brute en présence de $6 \mathrm{ml}$ de $\mathrm{KOH} 2 \mathrm{~N}$ selon la technique de Iiyama et al. [14], ce procédé permet d'extraire la quasi-totalité des deux acides. La réaction a été arrêtée par refroidissement des tubes qui ont été acidifiés avec du $\mathrm{HCl} 6 \mathrm{~N}$ jusqu'à un $\mathrm{pH}$ de 2,5 puis, $10 \mathrm{ml}$ d'acétate d'éthyle y ont été introduits. Après agitation, les tubes ont été centrifugés pendant 15 min à $4000 \mathrm{~g}$ en milieu réfrigéré ; le surnageant est récupéré et l'opération est répétée trois fois. Les trois surnageants réunis ont été séchés sous vide, et repris dans $2 \mathrm{ml}$ de méthanol et conservés à $-18^{\circ} \mathrm{C}$.

La séparation des deux acides a été réalisée sur un volume de $10 \mu \mathrm{l}$ à l'aide d'un analyseur HPLC de marque Waters, muni d'une colonne Beckman ultrasphère C18. L'éluent était composé de $7 \%$ d'acide for- mique dans du méthanol; le débit a été de $0,8 \mathrm{ml} / \mathrm{min}$ et la détection s'est faite à $310 \mathrm{~nm}$. L'identification des pics a été réalisée par comparaison à des standards d'acides $p$-coumarique et férulique et leur calcul par intégration automatique.

\subsection{Dégradation de l'azote dans le rumen}

La dégradabilité de l'azote a été mesurée par la technique des sachets de nylon, sur trois vaches Frisonnes munies d'une canule ruminale. Les animaux ont reçu deux fois par jour ( $8 \mathrm{~h}$ et $16 \mathrm{~h}$ ) dans les proportions 70-30, un foin de luzerne-dactyle et un concentré (dosant $15,4 \%$ de MAT) pour bovin composé de : $43 \%$ d'orge ; $40 \%$ de pulpes de betterave déshydratée ; $10 \%$ de tourteau de soja 44 ; $5 \%$ de mélasse de betterave ; $0,5 \%$ de sel et $1 \%$ de CMV.

Les sachets sont confectionnés dans un tissu Blutex T50 (maille de $46 \mu$, dimension $7,5 \times 15 \mathrm{~cm})$. Le poids $(3 \mathrm{~g})$ et la préparation de l'échantillon ont été ceux préconisés par Michalet-Doreau et al. [26]. Les incubations ont été réalisées selon une cinétique en six points $(2,4,8,16,24$ et $48 \mathrm{~h})$ à raison de six répétitions par vache et par point. Les sachets du rumen après chaque prélèvement ont été rincés à l'eau, puis congelés. Après décongélation, tous les sachets ont été de nouveau lavés à la machine ( 3 cycles de $5 \mathrm{~min}$ ), essorés pendant $8 \mathrm{~h}$ à $30^{\circ} \mathrm{C}$ ensuite lyophilisés pendant $24 \mathrm{~h}$ puis pesés. Sur l'azote dosé une correction de contamination bactérienne a été ajoutée, elle est égale à 6,4-0,365 MAT + 0,170 NDF [28].

Les données de la cinétique de la dégradation de l'azote ont été ajustées au modèle monomoléculaire de Ørskov et McDonald [30] pour calculer les paramètres a (fraction d'azote immédiatement dégradable) ; b (fraction d'azote potentiellement dégradable) et c (vitesse de dégradation de la fraction b). La dégradabilité théorique de l'azote $(\mathrm{dtN})$ est calculée par intégration du modèle : 
$\mathrm{dtN}=\mathrm{a}+\mathrm{b} \cdot \mathrm{c} / \mathrm{c}+\mathrm{k}$ avec un taux de renouvellement $(\mathrm{k})$ égal à $0,06 \mathrm{~h}^{-1}$ [37].

\subsection{Traitement statistique}

Les teneurs en parois, en MAT, en ApC, en $\mathrm{AF}$, les paramètres $\mathrm{a}, \mathrm{b}, \mathrm{a}+\mathrm{b}$ et $\mathrm{c}$ ainsi que la $\mathrm{dtN}$ et la dégradation de l'azote à $48 \mathrm{~h}$ (dG 48 h) ont été soumis à une analyse de variance en prenant en compte les effets : température, durée et dose d'ammoniac selon le modèle :

$$
\begin{gathered}
Y_{i j k l}=\mu+\alpha_{i}+\beta_{j}+\gamma_{1}+\alpha_{i} \beta_{j}+\alpha_{i} \gamma_{1}+\beta_{j} \gamma_{1} \\
+e_{i j k 1}
\end{gathered}
$$

où, $Y_{i j k l}$ représente la variable expliquée, $\mu$, la moyenne générale, $\alpha_{i}$ l'effet de la température, $\beta_{j}$ l'effet de la dose, $\gamma_{1}$ l'effet de la durée, $\alpha_{i} \beta_{\mathrm{j}}$, l'interaction température $\times$ dose, $\alpha_{i} \gamma_{1}$, l'interaction température $\times$ durée, $\beta_{j} \gamma_{1}$, l'interaction durée $\times$ dose, $e_{i j k l}$, l'erreur residuelle du modèle.

Par ailleurs, des régressions linéaires entre la teneur en ApC et en AF et la dégradabilité de l'azote ont été calculées.

L'ensemble des calculs a été réalisé avec le logiciel S-PLUS [31].

\section{RÉSULTATS}

\subsection{Composition chimique}

Comparativement à la paille non traitée (PNT), le traitement à l'ammoniac a amélioré logiquement la teneur en azote qui est passée de 2,1 à 11,3\% de la MS (Tab. I). Cette teneur en MAT a augmenté significativement $(p<0,01)$ avec la température de traitement. Les teneurs des différentes fractions de la paroi ont également été significativement modifiées par la température $(\mathrm{NDF}=79,4$ vs. $74,9 \%$ de la $\mathrm{MS}$; $\mathrm{ADF}=52,5$ vs. $46,1 \%$ de la MS ; $\mathrm{ADL}=8,1$ vs. $6,4 \%$ de la MS ; respectivement pour 15 et $35^{\circ} \mathrm{C}$ ).

Les teneurs en $\mathrm{ApC}$ et en $\mathrm{AF}$ ainsi que la somme $\mathrm{ApC}+\mathrm{AF}$ pour les 16 lots de paille traitée (PT) et pour le témoin PNT sont présentées dans le Tableau I. En moyenne, toutes pailles confondues, la somme ApC + AF a été égale à $0,7 \%$ de la MS pour la PNT et à $0,6 \%$ de la MS pour les PT, soit après traitement, une diminution significative de $16 \%(p<0,04)$. Cette diminution a été de $22 \%(p<0,001)$ pour AF et de $7 \%(p<0,05)$ pour ApC.

Au sein des PT, sous l'effet du traitement, la somme ApC + AF a varié de $0,5 \%$ pour PT14 à 0,7\% de la MS pour PT1 soit respectivement pour ApC et pour AF, des écarts extrêmes entre pailles de -29\% (PT1 et PT14) et $-48 \%$ (PT1 et PT16).

L'analyse de variance a montré que les variations de teneurs en ApC et en AF sont expliquées pour l'essentiel par la température de traitement (ApC, $p<0,03$; ApC + $\mathrm{AF}, p<0,05)$ tandis que, la teneur en $\mathrm{AF}$ est modifiée à la fois par les trois paramètres de traitement et leurs intéractions (Tab. I).

\subsection{Dégradabilité de l'azote des pailles, effet des conditions de traitement et des acides phénoliques}

La dtN de la PNT a été faible : $15 \%$. Sa valeur a augmenté tout à fait logiquement avec l'apport d'azote inhérent au traitement. En moyenne, la dtN a été plus faible pour les traitements à $15^{\circ} \mathrm{C}$ que ceux à $35^{\circ} \mathrm{C}: 63 \%$ vs. $71 \%$ (Tab. II). Cette augmentation a résulté de celle des paramètres a $(+15 \%$; $p<0,001)$, à un moindre degré de $\mathrm{b}(+5 \%$; $p<0,05)$ et surtout de $\mathrm{c}\left(+27^{\circ} \mathrm{C} ; p<0,001\right)$. La fraction potentiellement dégradable $a+b$ et dG $48 \mathrm{~h}$ ont présenté des valeurs comparables et ont été modifiés par les mêmes facteurs de traitement : température et dose d'ammoniac, alors que la fraction a, très variable (car très corrélée avec dg $2 \mathrm{~h}$ qui intègre une bonne partie des variations liées à l'incubation), a été sensible à la fois aux trois facteurs de traitement et à leurs interactions.

De façon générale, la dégradation de l'azote à chaque point de cinétique, la $\mathrm{dtN}$ et 
Tableau II. Effet des conditions de traitement sur la dégradabilité de l'azote.

\begin{tabular}{|c|c|c|c|c|c|c|}
\hline Pailles & $\operatorname{Dg} 48 \mathrm{~h}$ & $\mathrm{dtN}$ & $\mathrm{C}$ & $\mathrm{a}$ & $\mathrm{b}$ & $a+b$ \\
\hline PT1 & $86,2 \pm 2,0$ & $65,7 \pm 1,0$ & $0,17 \pm 0,02$ & $12,3 \pm 4,0$ & $73,6 \pm 4,1$ & $85,9 \pm 1,2$ \\
\hline PT2 & $83,6 \pm 1,2$ & $62,6 \pm 1,4$ & $0,16 \pm 0,03$ & $7,4 \pm 3,8$ & $75,02 \pm 3,5$ & $82,5 \pm 1,3$ \\
\hline PT3 & $86,2 \pm 0,7$ & $63,7 \pm 1,4$ & $0,15 \pm 0,01$ & $9,5 \pm 5,8$ & $75,8 \pm 4,9$ & $85,3 \pm 1,0$ \\
\hline PT4 & $83,4 \pm 0,8$ & $61,8 \pm 0,9$ & $0,13 \pm 0,02$ & $16,9 \pm 4,0$ & $65,7 \pm 3,8$ & $82,5 \pm 0,5$ \\
\hline PT5 & $81,8 \pm 0,5$ & $61,0 \pm 0,5$ & $0,15 \pm 0,03$ & $14,7 \pm 5,9$ & $65,1 \pm 5,2$ & $79,8 \pm 1,6$ \\
\hline PT6 & $82,1 \pm 0,9$ & $63,3 \pm 1,3$ & $0,16 \pm 0,02$ & $18,0 \pm 1,5$ & $63.8 \pm 3.2$ & $81,8 \pm 2,8$ \\
\hline PT7 & $78,9 \pm 1,1$ & $61,0 \pm 1,0$ & $0,18 \pm 0,00$ & $4,4 \pm 2,9$ & $78,7 \pm 2,6$ & $81,0 \pm 0,9$ \\
\hline PT8 & $80,4 \pm 1,7$ & $63,0 \pm 1,1$ & $0,21 \pm 0,02$ & $8,4 \pm 1,8$ & $68,8 \pm 1,7$ & $76,9 \pm 0,1$ \\
\hline РT9 & $88,1 \pm 2,1$ & $68,9 \pm 1,6$ & $0,18 \pm 0,02$ & $14,1 \pm 9,7$ & $72,1 \pm 9,5$ & $86,3 \pm 0,6$ \\
\hline PT10 & $89,5 \pm 0,8$ & $70,5 \pm 1,5$ & $0,25 \pm 0,03$ & $7,2 \pm 3,7$ & $78,4 \pm 3,4$ & $85,8 \pm 1,7$ \\
\hline PT11 & $91,6 \pm 1,1$ & $71,4 \pm 0,9$ & $0,20 \pm 0,02$ & $12,1 \pm 3,9$ & $77,6 \pm 2,8$ & $89,74 \pm 1,8$ \\
\hline PT12 & $89,9 \pm 1,3$ & $71,9 \pm 1,9$ & $0,22 \pm 0,03$ & $9,3 \pm 7,9$ & $78,0 \pm 8,8$ & $87,9 \pm 0,64$ \\
\hline PT13 & $88,7 \pm 0,5$ & $69,5 \pm 1,3$ & $0,16 \pm 0,01$ & $20,8 \pm 3,9$ & $65,8 \pm 2,9$ & $86,5 \pm 0,1$ \\
\hline PT14 & $86,9 \pm 2,2$ & $70,4 \pm 1,0$ & $0,18 \pm 0,03$ & $24,2 \pm 7,3$ & $60,4 \pm 5,6$ & $84,8 \pm 1,6$ \\
\hline PT15 & $89,9 \pm 0,2$ & $70,5 \pm 1,4$ & $0,21 \pm 0,04$ & $10,8 \pm 4,8$ & $77,0 \pm 4,5$ & $87,8 \pm 0,5$ \\
\hline PT16 & $89,5 \pm 1,4$ & $72,5 \pm 0,9$ & $0,26 \pm 0,06$ & $6,3 \pm 0,6$ & $81,3 \pm 4,2$ & $87,6 \pm 1,0$ \\
\hline PNT & $45,2 \pm 1,6$ & $15,5 \pm 2,6$ & $0,02 \pm 0,00$ & $-4,7 \pm 2,1$ & $98,5 \pm 10,8$ & $93,8 \pm 8,7$ \\
\hline $\mathrm{T}$ & $* * *$ & $* * *$ & $* * *$ & $* * *$ & * & *** \\
\hline D & $*$ & NS & NS & $* * *$ & NS & $*$ \\
\hline $\mathrm{d}$ & NS & NS & NS & $* * *$ & NS & NS \\
\hline $\mathrm{T} \times \mathrm{D}$ & NS & NS & NS & $* * *$ & NS & NS \\
\hline $\mathrm{T} \times \mathrm{d}$ & NS & NS & NS & $* * *$ & NS & NS \\
\hline $\mathrm{D} \times \mathrm{d}$ & NS & NS & NS & $* * *$ & NS & NS \\
\hline RSD & 1,52 & 1,37 & 0,012 & 0,59 & 1,96 & 1,50 \\
\hline $\mathrm{R}^{2}$ & 0,97 & 0,98 & 0,98 & 0,99 & 0,98 & 0,96 \\
\hline
\end{tabular}

$\mathrm{T}$ : température ; D : dose ; d : durée ; RSD : écart-type résiduel du modèle ; R2 : taux de variation expliquée du modèle ; NS : non significatif ; ${ }^{*}: p<0,05 ; * * *: p<0,01$ à 0,001 . La PNT, n'est pas prise en compte dans l'analyse de variance. La MS est dosée, immédiatement à la sortie des sachets.

ses paramètres $\mathrm{a}, \mathrm{b}$ et $\mathrm{c}$ ont eu de faibles corrélations avec $\mathrm{ApC}, \mathrm{AF}$ et $\mathrm{ApC}+\mathrm{AF}$ (Tab. III). Les coefficients de corrélation les plus élevés (moyenne : $-0,60 ; p<0,01$ ), ont été observés dans la zone $\mathrm{t} 2-\mathrm{t} 8 \mathrm{~h}$, au début de l'incubation pour $\mathrm{ApC}$ et $\mathrm{ApC}+\mathrm{AF}$. En revanche, $\mathrm{AF}$ n'a pas diminué significativement la dégradabilité de l'azote in situ.

\section{DISCUSSION}

Nos résultats concernant l'effet des différents traitements sur la composition en azote et en parois sont globalement confor- mes à ceux de la littérature, ils n'appellent pas de commentaires particuliers.

Aussi, dans cette discussion, insisterons nous sur l'effet des conditions de traitement sur la teneur des pailles en ApC et en AF ainsi que les relations entre ces deux acides et la dégradabilité de l'azote in situ.

\subsection{Teneur en ApC et en AF des pailles, effet des facteurs de traitement à l'ammoniac}

Les teneurs de la PNT en ApC et en AF, respectivement 0,29 et $0,39 \%$ de la $\mathrm{MS}$, 
Tableau III. Relations entre la dégradabilité de l'azote et leur teneur en acides $p$-coumarique et férulique.

\begin{tabular}{lccc}
\hline & ApC & AF & ApC + AF \\
\hline dG 2 H & $-0,71$ & $-0,52$ & $-0,65$ \\
& $* * *$ & $*$ & $* * *$ \\
& & & \\
dG 4 H & $-0,57$ & $-0,34$ & $-0,56$ \\
& $*$ & & $*$ \\
dG 8 H & $-0,54$ & $-0,37$ & $-0,50$ \\
& $*$ & & $*$ \\
dG 16 H & $-0,15$ & 0,17 & $-0,02$ \\
dG 24 H & 0,05 & 0,39 & 0,24 \\
dG 48 H & 0,06 & 0,34 & 0,20 \\
a & $-0,28$ & $-0,38$ & $-0,37$ \\
b & 0,05 & 0,24 & 0,15 \\
c & $-0,27$ & $-0,39$ & $-0,37$ \\
a + b & $-0,33$ & 0,013 & $-0,21$ \\
dtN & 0,55 & $-0,44$ & $-0,57$ \\
& $*$ & & $*$ \\
\hline
\end{tabular}

$*: p<0,05 ; * * *: p<0,01$ à $p<0,001$.

sont comparables à celles observées dans les pailles de blé par d'autres auteurs [18, $25]$.

Après traitement, la teneur des pailles en ces deux acides a diminué en moyenne de $22 \%(\mathrm{ApC}, p<0,001)$ et de $7 \%$ (AF, $p<0,05)$. Des diminutions de 13 à $50 \%$ pour $\mathrm{AF}$ et de 7 à $41 \%$ pour $\mathrm{ApC}$ sont rapportées dans la littérature $[11,25]$. La diminution de la teneur de ces deux acides dans les pailles traitées pourrait être liée aux modifications de structure qu'induit le traitement, notamment, la coupure des liaisons covalentes qui associent l'AF aux hémicelluloses et celle des liaisons ester et éther associant l'ApC et l'AF aux lignines [4, 15, 18], les liaisons ester étant plus labiles pour AF $[19,38]$. Ces acides s'associeraient alors avec d'autres corps (notamment avec l'azote du traitement) pour former des composés qui ne sont plus identifiables en tant que $\mathrm{AF}$ et ApC.

\subsection{Dégradabilité de l'azote et relation avec la teneur en $\mathrm{ApC}$ et en $\mathrm{AF}$}

La valeur de dégradabilité de l'azote des 16 pailles traitées s'établit en moyenne à $67 \%$; elle est comparable à celle relevée dans la littérature [11-13, 27] pour un protocole d'incubation comparable à celui que nous avons utilisé.

Exprimés par rapport à l'azote apporté par le traitement et pour un taux de renouvellement de $0,06 \mathrm{~h}^{-1}$ [37], $72 \%$ de cet azote quittent les sachets contre $86 \%$ en moyenne pour d'autres travaux [12, 27].

Les conditions de traitement, notamment la température, ont un effet sur la quantité de MAT quittant les sachets ; ainsi, avec les pailles traitées à $35^{\circ} \mathrm{C}, 71 \%$ des MAT de la paille $(8,7 \%$ de la MS) sont dégradés contre $63 \%(5,7 \%$ de MS) pour celles traitées à $15^{\circ} \mathrm{C}$.

Cette meilleure dégradation des PT $35^{\circ} \mathrm{C}$ $(p<0,001)$ s'explique probablement par une teneur en azote plus élevée des échantillons et une teneur moindre en parois. Il ressort de ces résultats que, pour l'essentiel et en accord avec d'autres études [12, 27], la thèse d'une fixation irréversible de l'azote des pailles ammoniaquées ne peut être retenue pour expliquer l'excrétion anormalement élevée d'azote fécal chez les animaux consommant ce type de fourrage.

En ce qui concerne, ApC et $\mathrm{AF}$, leur quantification dans l'extrait montre que leur présence est plus élevée dans les PT $15{ }^{\circ} \mathrm{C}$ $(p<0,01)$. Leur action dépressive (notamment pour ApC) sur l'activité microbienne du rumen est rapportée par de nombreux auteurs [1, 5, 22, 23, 38], ainsi que celle plus spécifique sur la $\mathrm{dtN}$ des fourrages $[8$, 16].

Dans notre étude, la dégradation de l'azote durant les 8 premières heures d'incubation ( $\mathrm{dG} 2 \mathrm{~h} ; \mathrm{dG} 4 \mathrm{~h}$ et $\mathrm{dG} 8 \mathrm{~h}$ ) et la dtN diminuent avec la teneur en ApC $(p<0,04$ à $p<0,001)$ avec des coefficients de corrélation respectifs variant de $-0,71$ à $-0,54$ et de $-0,65$ à $-0,54$ (Tab. III). L'action fugace 
de ApC sur la dégradation de l'azote dans les sachets s'expliquerait soit par un lessivage rapide dans le rumen ; certaines études estiment en effet que l'essentiel de ces acides quittent le sachet durant les 4 premières heures de séjour dans le rumen [17]; soit par conversion sous une forme moins toxique par hydrogénation $[21,23]$.

Dans notre étude, un tiers de la $\mathrm{dtN}\left(\mathrm{R}^{2}\right.$ variant entre 0,30 et 0,37 ) est expliqué par la présence de ApC. Ce résultat montre, que sa contribution à la diminution de la dégradabilité n'est pas négligeable même si on ne peut sur cette base impliquer fortement les acides phénoliques dans l'excrétion fécale azotée anormalement élevée. En supposant que la totalité de ces acides est libérée dans le rumen d'un ovin, leur concentration ne représenterait que $0,1 \%$ contre des seuils de toxicité variant pour les auteurs entre $0,1 \%$ [1] et $2 \%$ [5].

Une hypothèse plus pertinente serait que les 12 à $15 \%$ de composés phénoliques des pailles [9] formeraient avec l'ammoniac apporté par le traitement, des complexes azotés solubles et indigestibles [35]. Ces complexes, libérés dans le rumen emprisonneraient de façon irréversible une partie de l'azote provenant du traitement qui viendrait ainsi enrichir les fèces. Ceci pourrait expliquer que certains auteurs [7, 10], notent que seulement la moitié de l'azote qui disparaît in sacco se retrouve sous forme d'ammoniac dans le rumen.

L'utilisation d'ammoniac ou d'acides phénoliques marqués permettrait de mieux étudier ce processus.

\section{CONCLUSION}

Le traitement à l'ammoniac diminue la teneur des pailles en acides $p$-coumarique et férulique respectivement de $7 \%$ et de $22 \%$. En revanche, il augmente la dégradabilité de l'azote total qui s'établit en moyenne à $67 \%$; elle varie de $63 \%$ (paille traitée à $15{ }^{\circ} \mathrm{C}$ ) à $71 \%$ (paille traitée à $35^{\circ} \mathrm{C}$ ). Exprimé sur la base de l'azote apporté par le traitement, $72 \%$ de cet azote quitte les sachets. Il n'est donc pas fixé de façon irréversible.

Les relations entre la dégradabilité de l'azote et les teneurs en acides $p$-coumarique et férulique, sans être très étroites, montrent que 30 à $37 \%$ de la dégradabilité de l'azote sont expliqués par la présence des deux acides. Ces valeurs ne sont pas négligeables même s'il est difficile encore ici de les impliquer fortement dans la faible digestibilité de l'azote des pailles traitées à l'ammoniac. D'autres hypothèses méritent d'être explorées, notamment, la formation dans les pailles traitées de composés azotés solubles mais indigestibles.

\section{REMERCIEMENTS}

Nous remercions le laboratoire INRA d'amélioration des plantes de Dijon pour son aide dans le dosage des composés phénoliques.

\section{RÉFÉRENCES}

[1] Akin D.E., Forage cell wall degradation and p-coumaric, ferulic and sinapic acids, Agron. J. 74 (1982) 424-442.

[2] AOAC, Official methods of analysis, 12th edn.,Washington, DC, 1975.

[3] Benahmed H., Dulphy J.P., Note sur la valeur azotée de fourrages pauvres traités par l'urée ou par l'ammoniac, Ann. Zootech. 34 (1985) 335-346.

[4] Besle J.M., Cornu A., Jouany J.P., Roles of structural phenylpropanoids in forage cell wall digestion, J. Sci. Food Agric. 64 (1994) 171-190.

[5] Borneman W.S., Akin D.E., Vaneseltine W.P., Effect of phenolic monomers on ruminal bacteria, Appl. Environ. Microbiol. 52 (1986) 1331-1339.

[6] Chenost M., Dulphy J.P., Amélioration de la valeur alimentaire (composition, digestibilité, ingestibilité) des mauvais foins et des pailles par différents traitements, in : Demarquilly C. (Ed.), Les fourrages secs : Récolte, traitement et utilisation, INRA Paris, 1987, pp. 199-230.

[7] Dryden G.Mc.L., Kempton T.L., Digestion of organic matter and nitrogen in ammoniated barley straw, Anim. Feed Sci. Technol. 10 (1986) $65-75$. 
[8] Gabrielsen B.C., Vogel K.P., Anderson B.E., Ward J.K., Alcali labile cell wall phenolic and forage quality in switchgrass selected for differing digestibility, Crop Sci. 30 (1990) $1313-1320$

[9] Galletti G.C., Piccaglia V., Concialini M.T., Lippoli S., Determination of phenolic compounds from Lignocellulosic by-products by HPLC with electrochemical detector, in: Chesson A., Ørskov E.R. (Eds.), Physico-chemical characterisation of plant residues for industrial and feed use, CEE, 1988, pp. 131-139.

[10] Gordon A.H., Chesson A., The effect of prolonged storage on the digestibility and nitogen content of ammonia treated barley straw, Anim. Feed Sci. Technol. 8 (1983) 147-153.

[11] Graham H., Aman P., Maguire M.H., Influence of anhydrous ammonia treatment on the composition and degradation of components of barley straw, Ir. J. Agric. Res. 24 (1985) 33-37.

[12] Hassen L., Chenost M., Tentative explanation of the abnormally high faecal nitogen-excretion with poor quality roughages treated with ammonia, Anim. Feed Sci. Technol. 38 (1992) 25-34.

[13] Hvelplund T., Protein evaluation of treated straw, in: Chenost M., Reiniger P. (Eds.), Ruminant evaluation of straw in ruminant feeding, Elsevier Applied Science, 1989, pp. 66-74.

[14] Iiyama K., Lam T.B.S., Stone B.A., Phenolic acids bridges beween polysaccharides and lignin in wheat internodes, Phytochem. 29 (1990) 733-737.

[15] Jung H.G., Forage lignins and their effects on fiber digestibility, Agron. J. 81 (1989) 33-38.

[16] Komprda T., Zelenka J., Tvrznik P., Nedbalkova M., Variability sources of crude protein and organic matter degradability values measured in situ for testing the dependance of nutritive value of lucerne on the stage of maturity, J. Anim. Physiol. Anim. Nutr. 70 (1993) 190-195.

[17] Komprda T., Frantisek J., Stohandlova M., Edltyn J., In Situ disappearance and content of $p$-coumaric and ferulic acid in lucerne from various vegetative stages, Anim. Feed Sci. Technol. 67 (1997) 141-150.

[18] Kondo T., Ohshita T., Kyuma T., Comparison of characteristics of soluble lignins from untreated and ammonia-treated wheat straw, Anim. Feed Sci. Technol. 39 (1992) 253-563.

[19] Kondo T., Ohshita T., Kyuma T., Comparison of phenolic acids in lignin fractions from forage grasses before and after digestion by sheep, Anim. Feed Sci. Technol. 47 (1994) 277-285.

[20] Laurent F., Hasna J., Vignon B., Mesure par la méthode des sachets mobiles du taux de disparition de la matière sèche et de l'azote des contenus intestinaux chez des chèvres recevant des pailles traitées chimiquement, Ann. Zootech. 41 (1992) 25-26.
[21] Lowry J.B., Sumpter E.A., Mc Sweeney C.S., Schlink A.C., Bowden B., Phenolic acids in the fibre of some tropical grasses; effect on feed quality and their metabolism by sheep, Aust. J. Agric. Res. 44 (1993) 1123-1133.

[22] Martin S.A., Akin D.E., Effect of phenolic monomers on the growth and $\beta$-glucosidase activity of Bacteroides ruminicola and on the carboxymethylcellulase, $\beta$-glucosidase and xylanase activity of bacteroides succinnogènes, Appl. Environ. Microbiol. 54 (1988) 3019-3022.

[23] Marvin H.J.P., Cornelis F.K., Eibertus N.L., Charles H.A.S., Lommen A., Dolstra O., Relationship between phenolic acids formed during rumen degradation of maize samples and in vitro digestibility, J. Sci. Food Agric. 71 (1996) 111-118.

[24] Mason V.C., Cook J.E., Cooper E.M., Hoadley C.J., Cockburn J.E., Oven and stack ammoniation of grass hays. 2-Volontary intake, digestibility and faecal nitrogen excretion in sheep in relation to hay composition, Anim. Feed Sci.Technol. 24 (1989) 313-326.

[25] Mason V.C., Dhanoa M.S., Hartley R.D., Relationship between chemical composition digestibility in vitro and cell wall degradability of wheat straw treated with different amounts of ammonia and water at elevated temperature, Anim. Feed Sci. Technol. 27 (1990) 293- 306.

[26] Michalet-Doreau B., Vérité R., Chapoutot R., Méthodologie de mesure de la dégradabilité insacco de l'azote des aliments dans le rumen, Bull. Tech. C. R. Z. V. Theix 69 (1987) 5-7.

[27] Michalet-Doreau B., Guedes C.V.M., Influence du traitement des fourrages à l'ammoniac sur leur dégradabilité dans le rumen, Ann. Zootech. 38 (1989) 259-268.

[28] Michalet-Doreau B., Aufrère J., Nouvelles méthodes d'estimation de la valeur alimentaire des fourrages.1.Dégradabilité in sacco, Fourrages 122 (1990) 189-201.

[29] Oji U.I., Mowat D.N., Winch J.E., Alkali treatment of corn stover to increase nutritive value, J. Anim. Sci. 44 (1977) 798-802.

[30] Ørskov E.R., Mc Donald I., The estimation of protein degradability in the rumen from incubation measurements weighted according to rate of passage, J. Agric. Sci. (Camb.) 92 (1979) 499-503.

[31] S. Plus Statistics, 4-5e éd., Tokyo, 1998.

[32] Sundst $\varnothing 1$ F., Coxworth E.M., Mowat D.N., Improving the nutritive value of straw and other low quality roughages by treatment with ammonia, World Anim. Rev. FAO 26 (1978) 13-21.

[33] Sundst $ø 1$ F., Coxworth E.M., Ammonia Treatment, in: Sundstøl F., Owen E. (Eds.), Straw and other fibrous by-products as feed, Elsevier, Amsterdam, 1984, pp. 196-247. 
[34] Van Soest P.J., Use of detergents in the analysis of fibrous feeds. II. a rapid method for the determination of fiber and lignin, AOAC 46 (1963) 829-835.

[35] Van Soest P.J., Mason V.C., The influence of the Maillard reaction upon the nutritive value of fibrous feeds, Anim. Feed Sci. Technol. 32 (1991) 45-53.

[36 Varel V.M., Jung H.J., Influence of forage phenolic on ruminal fibrolytic bacteria and in vitro fiber degradation, Appl. Environ. Microbiol. 52 (1986) 275-280.

[37] Vérité R., Michalet-Doreau B.,Chapoutot P., Peyraud J.L., Poncet C., Révision du système des protéines digestibles dans l'intestin (PDI), Bull. Tech. C. R. Z. V. Theix 70 (1987) 19-34.

[38] Wilson J.R., Hatfield R.D., Structural and chemical changes of cell wall types during stem development: Consequences for fibre degradation by rumen microflora, Aust. J. Agric. Res. 48 (1997) 165-180. 